\title{
Ơkologische Untersuchungen an einem marinen Pilz aus der Umgebung von Helgoland
}

\author{
Alwin Gaertner \\ Institut für Meeresforschung, Bremerhaven
}

\begin{abstract}
Ecological investigations on a marine fungus from the area near Helgoland. A new fungus Thraustochytrium kinnei $n$. sp. was isolated for the first time from tidal currents between the island Helgoland and its nearby dune. It is characterized by a habitus and life cycle different from those of related species previously described. These differences allow for an easy identification and thus make the new species a suitable object for ecological studies on lower marine fungi. The presence of lower fungi from the genera Thraustochytrium, Schizochytrium and Dermocystidum was recorded at 5 permanent stations in the Weser estuary and the German Bight. T. kinnei was found during the period from July to December and only near Helgoland. Its restricted distribution seems to indicate that the new species is a littoral form which is transported in the form of zoospores in the tidal currents. Further investigations on the physiology of T. kinnei are presently being carried out.
\end{abstract}

\section{EINLEITUNG}

Während eines Aufenthaltes an der Biologischen Anstalt Helgoland im August 1963 konnte neben einer Mehrzahl mariner niederer Pilze eine Form aus dem Wasser des Gezeitenstromes zwischen Insel und Düne isoliert werden, welche sich im Verlaufe daran anschließender Untersuchungen als bisher noch nicht beschrieben erwies. Dieser Pilz, eine stark abgeleitete Form aus der Ordnung der Saprolegniales, in seinem Entwicklungsgang monocentrisch, das heißt, der Körper der Zoospore bildet sich in seiner Gesamtheit in das Sporangium um, gehört der Familie der Thraustochytriaceae an. Er wurde durch GAERTNER (1964) bereits zu elektronenoptischen Untersuchungen der Begeißelung seiner Zoosporen herangezogen, als es gelang, ihn unter Ausschluß von Bakterien zu kultivieren. Die Kultur ging dann später infolge chemischer Einflüsse verloren und konnte mit Aufnahme unserer Arbeiten zur Pilzdichte im Oktober 1964 aus der Umgebung von Helgoland wieder isoliert werden. Die Biologie und Okologie des Pilzes gibt dem sich damit Befassenden immer noch eine Reihe ungelöster Rätsel auf, so daß es zweckmäßig erscheint, den Pilz Ihnen in einer Studie zum Habitus und Entwicklungsgang im ersten Teile dieses Referates vorzustellen. 


\section{ERGEBNISSE UND DISKUSSION}

Der Organismus wurde nach der durch Harder (1948), HöHnK (1955, 1957), Sparrow (1960), Goldstein $(1963,1966)$, Gaertner $(1954,1966)$ und anderen vielseitig erprobten Ködermethoden aus dem Wasser isoliert, wobei Pollen von Pinus als Köder- und Kultursubstrat verwendet wurde. Hierbei zeigten sich in den ersten Ansätzen der entnommenen Wasserproben an einzelnen Pollenkörnern kleine Bläschen (Abb. 1), umgeben von einer feinen, kaum sichtbaren Hülle hyalinen Materials, die im späteren Verlaufe der Entwicklung zu den Zoosporangien (Abb. 2, 3) heranreifen. Die Zoosporangien sind meist birnenförmig, mit dem verjüngten Ende am Pollenkorn inseriert und zeichnen sich in ihrem Innern durch Unterteilung in einen basalen Körper und den darüberliegenden, sich in Zoosporen differenzierenden Teil aus (Abb. 2, 3). Im Zuge der weiteren Entwicklung wird diese Unterteilung in Plasmaportionen deutlicher, der obere, kalottenförmige Teil nimmt an Umfang etwas zu (Abb. 4, 5), und plötzlich reißt die inzwischen sehr fein gewordene Membran auf (Abb. 6), die Zoosporen werden frei und schwimmen in großer Eile davon (Abb. 7). Zurück bleibt, manchmal noch mit Anhängen von Teilen der Sporangienmembran besetzt, der im Innern entwickelte Restkörper (Abb. 8, 9), welcher sich unter günstigen Bedingungen zu einem neuen Sporangium ausdifferenzieren kann. Hierdurch wird das Sporangium in die Lage versetzt, zu proliferieren. Diese neuen Sporangien sind jetzt mehr oder weniger elliptisch, mit der Breitseite inseriert (Abb. 10) und bilden erneut einen Restkörper in ihrem Innern. Dieses Spiel wiederholt sich so lange, bis das Substrat erschöpft ist. Dann löst sich der letzte Restkörper häufig als selbständiges Gebilde aus seiner Membran (Abb. 11), kann aber auch an der alten Stelle liegen und mit dem Rhizoid verbunden bleiben.

Die Zoosporen, bohnenförmig von Gestalt, in sich abgeplattet, mit zwei nahezu gleichlangen, ungleich gebauten Geißeln (GAERTNer 1964), von denen die erste als Flimmergeißel, die andere als Peitschengeißel ausgebildet ist, sind sofort nach Aufreißen der Zoosporangiummembran dazu in der Lage, ohne Zwischenschaltung eines diplanetischen oder Ruhestadiums, das Substrat aufzusuchen und zu besiedeln. Sie setzen sich an dem ihnen zusagenden Material, im vorliegenden Falle am Pollenkorn, fest, treiben ein anfangs gerades, später auch verzweigtes Rhizoid in dasselbe hinein, mit dessen Hilfe sie sich dort verankern und die Nährstoffe aus dem Substrat herauslösen (Abb. 12). Dieses Zellorganell ist im späteren Verlaufe der Entwicklung, wenn es wieder plasmaleer geworden ist, nur unter günstigsten optischen Bedingungen sichtbar. Aus dem Körper der Zoospore wird so das Ihnen eingangs beschriebene Sporangium, welches dann seine Zoosporen entläßt.

Andert sich mit zunehmendem Alter der Kultur das Medium, sei es durch Erschöpfung bestimmter Stoffe, sei es durch Anreicherung dem Pilze eigener Stoffwechselendprodukte, so unterbleibt der Schwärmakt und die sichtbare Ausbildung des Restkörpers, und eine Erscheinung tritt in höherem Maße hervor, welche im frühen Stadium der Entwicklung bereits angelegt worden war: Die Sporangien umgeben sich mit einer dicken, hyalinen Membran, welche zunehmend stärker wird, und die gegebenenfalls sogar als geschichtete Hülle in Erscheinung tritt (Abb. 13, 14). Andern wir nun die Umgebung, indem wir solche Sporangien in frisches Kultursubstrat überfüh- 

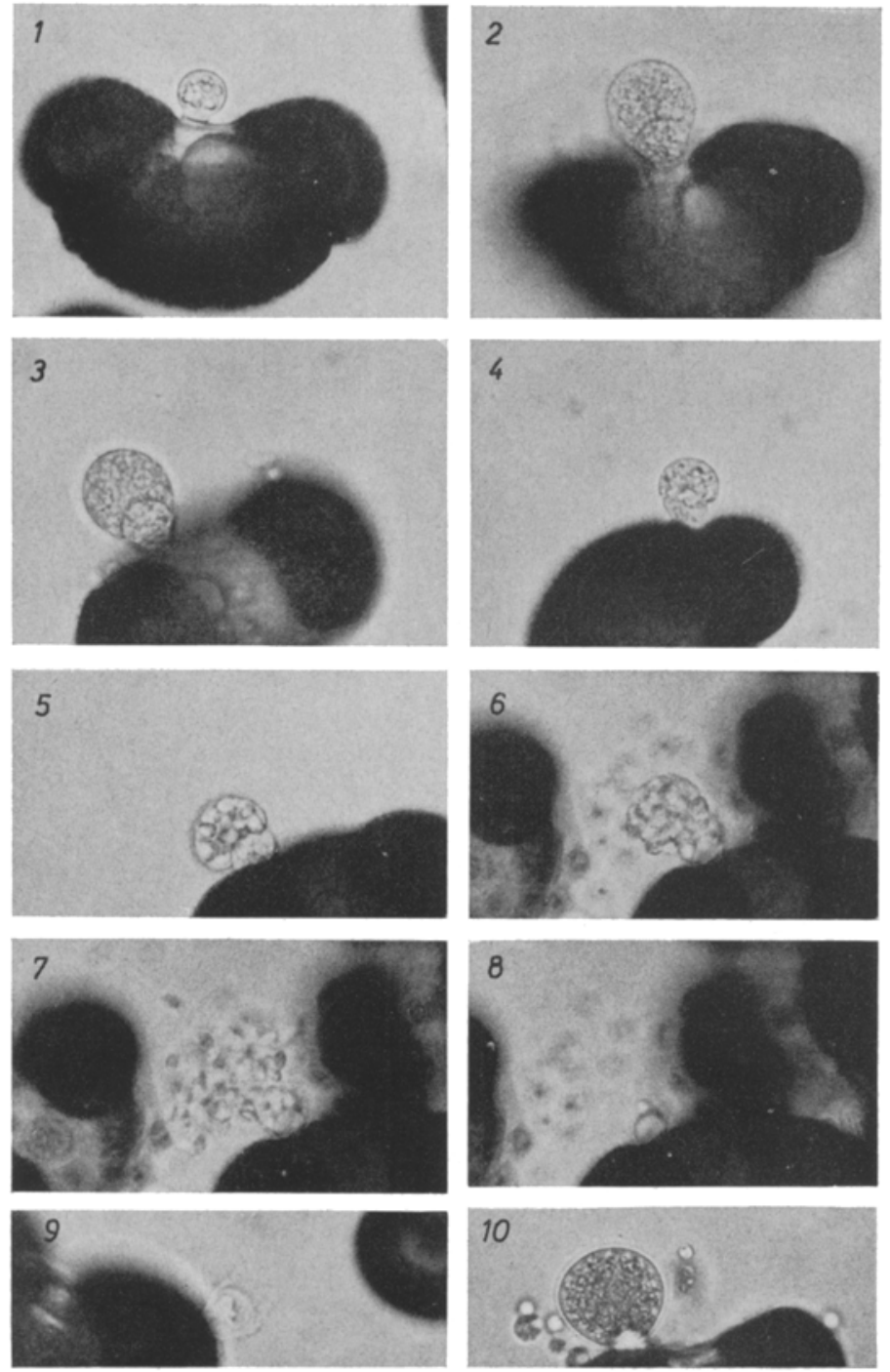

Abb. 1 bis 10: Entwicklung von Thraustochytrium kinnei n. sp. 1: Junger Thallus; erste Stadien seiner Entwicklung. 2: Spätere Stadien seiner Entwicklung; beginnende Umwandlung in Zoosporangien; erste sichtbare Abgliederung des Restkörpers; beginnende Aufteilung in Plasmaportionen. 3: Volle Ausdifferenzierung des Restkörpers. 4: Beginnende Ausdifferenzierung der Zoosporen im oberen Teile; anschwellen desselben. 5: Fortgeschrittene Ausbildung der Zoosporen; kurz vor dem Ausschwärmen. 6 bis 8: Schwärmakt; drei aufeinanderfolgende Stadien des gleichen Objekts. 6: Aufreißen der Zoosporangienmembran. 7. Die Zoosporen schwärmen sofort aus. 8: Der zurückgebliebene Restkörper mit einer noch daran haftenden Zoospore und Teilen der Zoosporangienmembran. 9: Destruierter Restkörper mit sichtbarer ehemaliger Zoosporangiummembran. 10: Proliferiertes Zoosporangium mit Teilen der alten Membran 
ren, so differenziert sich das Plasma in Zoosporen, welche den normalen Propagationszyclus vollziehen. Ferner können, sporadisch oder unter den Bedingungen zunehmenden Alters der Kultur, Stadien entstehen, welche bei anderen Vertretern der Thraustochytriaceae wesentlich häufiger vorkommen, gegebenenfalls sogar die Regel sind. Hier werden vom Hauptteile des Vegetationskörpers zahlreiche Nebenkörper abge-
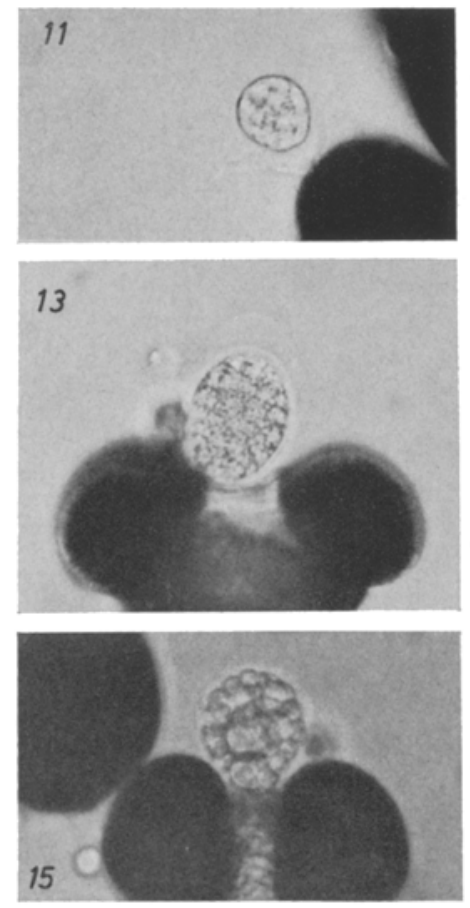
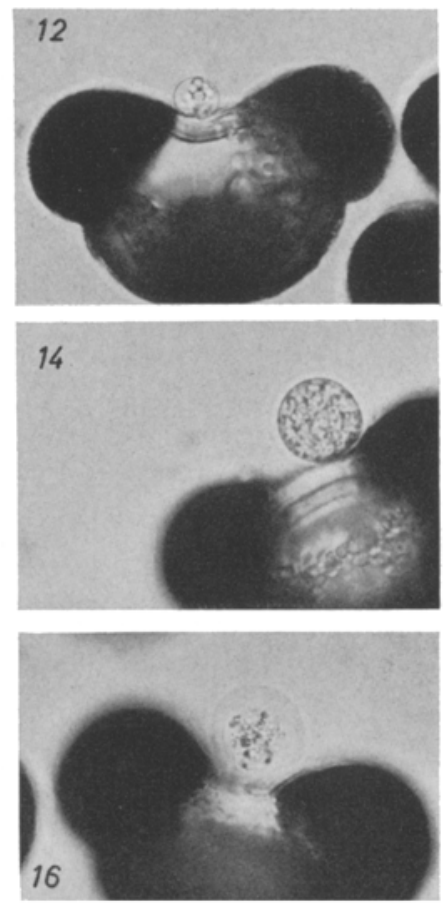

Abb. 11 bis 16: Weitere Entwicklung von $T$. kinnei. 11: Sich aus der alten Membran lösender Restkörper. 12: Junger Thallus mit sichtbarem, geradem Rhizoid. 13: Stagnierende Entwicklung; der Thallus hat eine dicke Membran ausgebildet. 14: Beginn der Verdickung einet solchen Membran. 15: Aufteilung des Thallus in selbständige Portionen, umgeben von einer eigenen Membran; jede dieser Portionen fungiert als selbständiges Sporangium. 16: Sporangium in Autolyse; dicke, geschichtete Membran

gliedert, die, von einer gemeinsamen hyalinen Hülle umgeben, jetzt ein vielzelliges Gebilde vortäuschen (Abb. 15). Von diesen fungiert jeder einzelne nach dem Übertragen in frisches Kultursubstrat als Zoosporangium.

Bei einem längerdauernden Verweilen in dem Erschöpfungszustande schreitet die Verdickung der Membran weiter fort, bis der Organismus degeneriert und abstirbt. Ein solches Stadium zeigt Abbildung 16.

Dieser Pilz mit seinen proliferierenden Sporangien, aber mit sofort beweglichen Zoosporen, steht zwischen den schon länger bekannten Arten, dem proliferierenden Thraustochytrium proliferum SPARROW (1936), bei welchem jedoch die Zoosporen ein Ruhestadium durchmachen, JoHnson (1957) und Thraustochytrium globosum Ko- 
вayasi \& Oокubo $(1953,1954)$, bei dem die Proliferation gänzlich fehlt, aber die Zoosporen sofort beweglich sind. Es kann somit auch auf Grund seiner morphologischen Verschiedenheiten JoHNSON (1957) der Gattung Thraustochytrium als neue Art hinzugefügt werden, welche als als Bindeglied zwischen diesen beiden schon länger bekannten Arten steht.

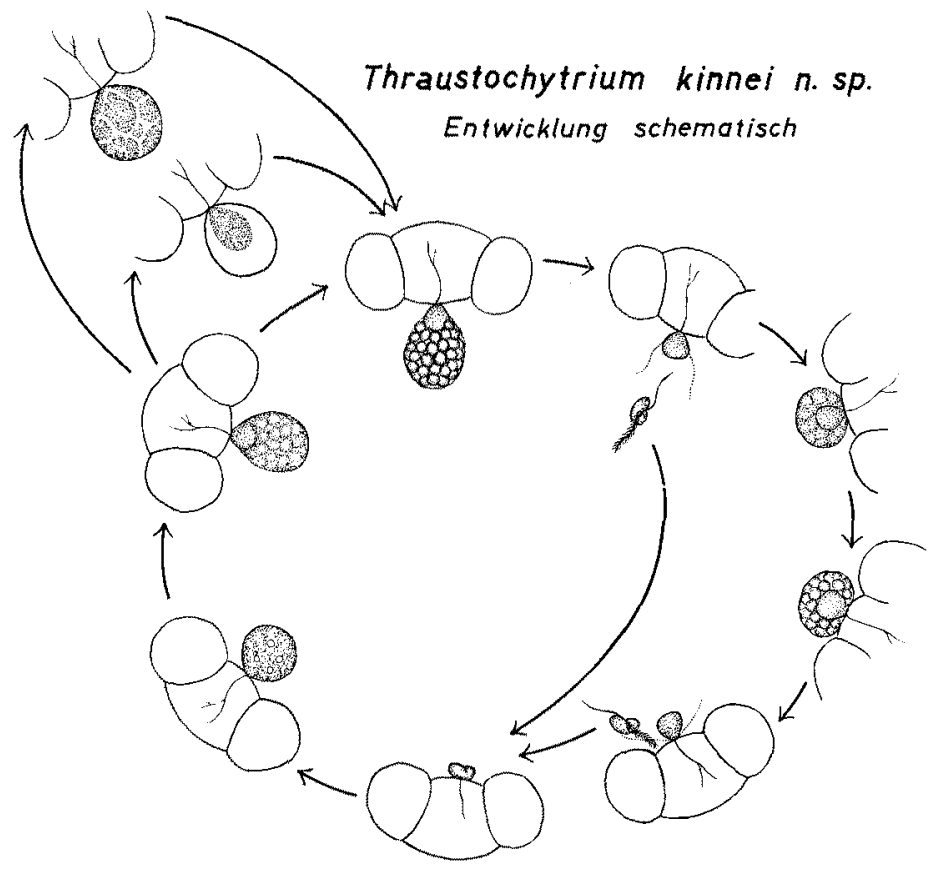

Abb. 17: Lebenszyklus von Thraustochytrium kinnei n. sp. (schematisch)

Zu Ehren von Herrn Prof. Dr. O. Krnne, welcher zum Auffinden des Pilzes durch freundliches Bereitstellen eines Arbeitsplatzes und der Einrichtungen der Biologischen Anstalt Helgoland während meines Urlaubs im Jahre 1963 maßgeblich beigetragen hat, sei der beschriebene Pilz Thraustochytrium kinnei n. sp. benannt. Der Entwicklungsgang ist in Abbildung 17 dargestellt.

\section{Diagnose}

Thraustochytrium kinnei $\mathrm{n}$. sp.

Mariner Pilz. Thallus monocentrisch, epi- und endobiontisch, bestehend aus dem birnenförmigen Sporangium $(14-17 \times 17-19 \mu)$ mit dem verjüngten Ende am Substrat inseriert und dem sehr dünnen, anfangs geraden, später verzweigten Rhizoid im Substrat verankert. Bei der Reife differenziert sich das Plasma in einen $10 \mu$ großen 
Restkörper, am verjüngten Ende des Sporangiums liegend, und den Zoosporen, welche diesem kalottenförmig aufsitzen. Entlassen der Zoosporen nach Aufreißen der Membran an einer oder mehreren Stellen. Das Sporangium proliferiert aus dem Restkörper. Dieses Sporangium ist elliptisch in Form $(16 \times 14 \mu)$ mit der Breitseite inseriert. Entlassung der Zoosporen wie vorher. Zoosporen 4,5 bis $4,8 \times 2,5$ bis $3 \mu$ groß, bohnenförmig, abgeplattet mit einem etwas größeren Inhaltskörper am hinteren Ende und mehreren Grana besitzen zwei seitlich inserierte, nahezu gleichlange Geißeln (12 $\mu$ lang) und bewegen sich sehr schnell gleitend oder pendelnd durch das Wasser. Geißeln unterschiedlich gebaut, davon die eine nach dem Flimmer-, die andere nach dem Peitschentypus. Mit zunehmendem Alter der Kultur tritt eine Verdickung der hyalinen, teilweise auch geschichteten Membran der Vegetationskörper ein (bis zu $6 \mu$ dick). Diese Vegetationskörper verhalten sich nach Änderung der Bedingungen wieder wie Sporangien. Vorkommen in der Uferzone um Helgoland, köderbar mit Pinuspollen. Substrat unbekannt.

\section{Thraustochytrium kinnei $\mathrm{n} . \mathrm{sp}$.}

Fungus marinus. Thallus monocentricus, epi- et endobioticus, constat ex sporangio piriformi $(14-17 \mu \times 17-19 \mu)$, parte conica substrato inserto, et ex rhizoido tenuissimo, primum recto, postea late diffuso substrato penitus insito. Maturescens plasma discedit in corpus reliquum $(10 \mu$ diametro) in parte conica sporangii et in zoospores qui corpori adhaeret. Zoospores dimittuntur membrana rupta uno loco aut pluribus locis. Sporangium ex corpore reliquo prolifert. Hoc sporangium forma elliptica $(16 \times 14 \mu)$ parte lata insertum est. Zoospores dimittuntur modo antea descripto. Zoospores $4,5-4,8 \times 2,5-3 \mu$ in latitudinem, forma fabae, plani cum corpore interiore (= Inhaltskörper) paulum maiore ad partem aversam et cum pluribus granis duo flagellas gerunt prope aequali longitudine lateribus insertas (12 $\mu$ longitudine). Celerrime per aquam moventur labentes vel nutantes. Flagellae differunt forma, alterae cum filis scintillantibus, alterae sicut flagra. In cultura vetustescente membranae corporum vegetationis hyalinae, partim structae crassiores fiunt (ad $6 \mu$ ). Haec corpora vegetationis mutata conditione denuo se gerunt sicut sporangia. Reperitur in litore circum Helgoland, pollinibus pini inescatur. Substratum ignotum est.

\section{Okologische Untersuchungen}

Im Rahmen eines Forschungsvorhabens führen wir seit dem 1. Oktober 1964 vom Institut für Meeresforschung aus an fünf Dauerstationen von der Wesermündung bis in die Deutsche Bucht über Helgoland hinaus laufend Untersuchungen zur Pilzbesiedlung des Meerwassers in quantitativer Hinsicht durch, wobei wir versuchen, nicht nur die Gesamtzahl der vorkommenden Niederen, Pollen besiedelnden Pilze zu erfassen, sondern auch bemüht sind, Einsichten in die Artenspektren zu gewinnen. Letzteres stößt auf die jedem mit mikrobiologischen Methoden Vertrauten bekannten Schwierigkeiten, besonders arbeitstechnischer Art und erfordert durch die Notwendigkeit, große Zahlen von Einzelisolaten anfertigen $\mathrm{zu}$ müssen, einen ungewöhnlich 
hohen Einsatz von Arbeitskraft. Dies besonders deshalb, weil die ganze Gruppe der Pollen besiedelnden Pilze, vor allem die Thraustochytrien, Schizochytrien und Dermocystidien auf geringfügige Veränderungen ihrer Umwelt mit einer großen Variabilität ihrer Gestalt antworten, so daß letzten Endes nur artreine Kulturen, welche aus einem oder einigen wenigen gleichgestalteten Individuen herangezogen wurden, Aufschluß über die Zusammensetzung einer Mischpopulation geben können. Hiervon macht Thraustochytrium kinnei n. sp. durch seine charakteristischen, verhältnismäßig leicht unterscheidbaren Stadien eine Ausnahme, so daß sich dieses Objekt für eine erste Auswertung der Artenzusammensetzung anbot.

Die Untersuchung der Pilzdichte wurde nach dem durch Gaertner (1966) in Anlehnung an die MPN-Methode entwickelten Verfahren durchgeführt. Es beruht auf dem Prinzip, Verdünnungsreihen mit sterilisiertem Seewasser der zu testenden Entnahme herzustellen, welche mit Pollen beködert nach frühestens 14 Tagen auf die

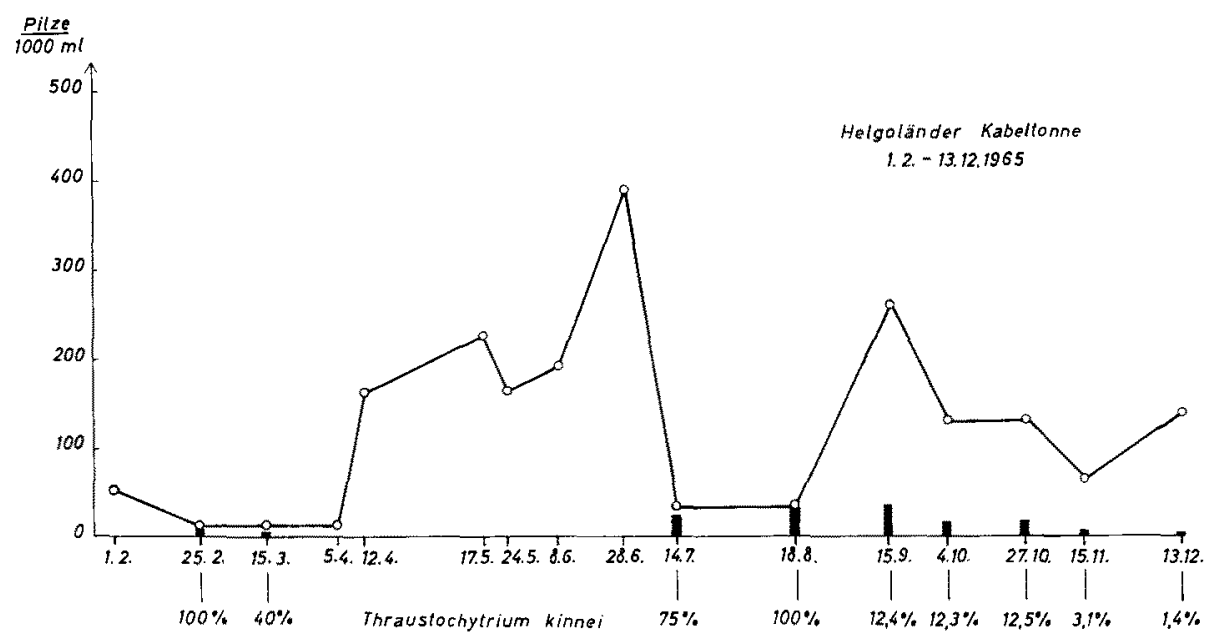

Abb. 18: Fluktuation Niederer, Pollen besiedelnder Pilze an der Dauerstation Helgoland Kabeltonne vom 1. Februar bis 13. Dezember 1965. T. kinnei als Säulendiagramm dargestellt. Darunter die Werte des prozentualen Anteiles von $T$. kinnei

diesen besiedelnden Pilz mikroskopisch untersucht werden. Das Verfahren arbeitet sehr genau und kann - sofern niedrige Besatzdichten erwartet werden - durch entsprechend abgestufte Reihen größerer Quantitäten unverdünnten Seewassers beliebig erweitert werden. Darüber hinausgehend konnte es nach Uberwindung einiger Schwierigkeiten auch für die Ermittlung des Pilzbesatzes in Sedimenten eingesetzt werden, über die noch zu einem späteren Zeitpunkt berichtet werden wird.

In Abbildung 18 wird der Besatz mit Niederen Pilzen an unserer Dauerstation "Helgoland Kabeltonne" für die Zeit vom 1. Februar bis 13. Dezember 1965 als Kurve dargestellt. Im Säulendiagramm ist gleichzeitig der Anteil von Thraustochytrium kinnei $\mathrm{n}$. sp. bei den insgesamt 16 Fahrten des Jahres 1965 eingezeichnet.

Im Monat Februar setzte sich ein Abfall der Häufigkeit Niederer Phycomycetes 
fort, welcher mit sehr hohen Besatzdichten von über 2200 Pilzeinheiten je Liter Seewasser im ausgehenden Jahre 1964 sein Maximum gehabt hatte. Am 25. Februar war das Minimum der Verteilung erreicht und hielt sich dann mit 10 Einheiten/l Seewasser konstant, um zwischen dem 5. April und 12. April innerhalb von sieben Tagen auf

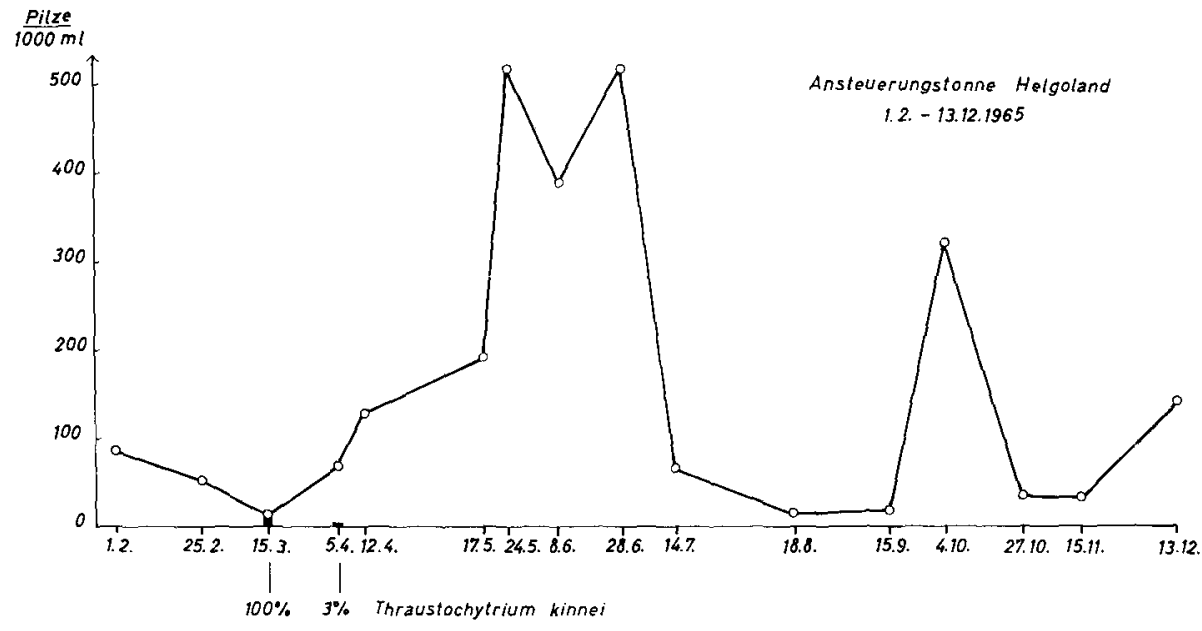

Abb. 19: Dauerstation Helgoland Ansteuerungstonne. (Wie Abb. 18)

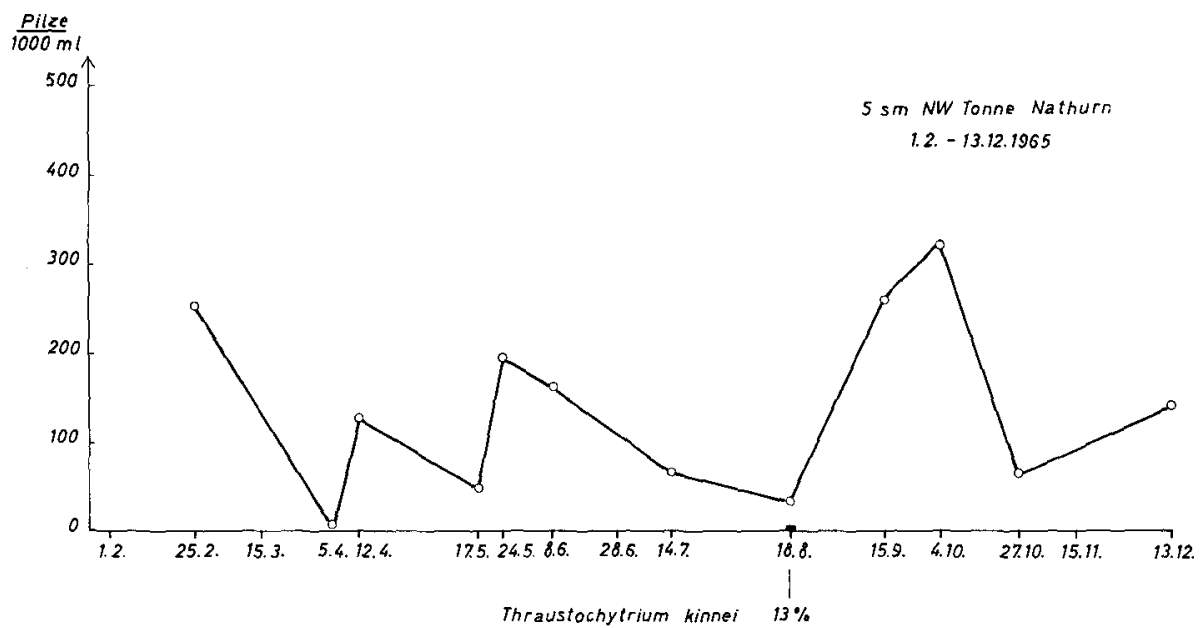

Abb. 20: Dauerstation 5 sm NW Tonne Nathurn. (Wie Abb. 18)

das 15 fache anzusteigen. Nach einigen geringfügigeren Fluktuationen erreicht der Besatz am 18. Juni mit nahezu 400 Einheiten je Liter den höchsten Wert, um dann am 14. Juli auf einen erneut niedrigen Stand von 32 Pilzen je Liter abzusinken. Dieser Stand war auch noch am 18. August, also vier Wochen später, vorhanden. Am 15. September erreichte er dann eine erneute Spitze, die unter Fluktuationen am Ende des Jahres wieder abklang. 
Betrachten wir zu diesem allgemeinen Pilzverlauf den Besatz mit Thraustochytrium kinnei $\mathrm{n}$. sp., so fällt generell auf, daß sein Vorkommen offensichtlich nicht an das Vorkommen und an den Ablauf der übrigen niederen Vertreter gebunden ist, sondern daß hier andere Faktoren ursächliche Auswirkungen haben müssen. So fehlte Thraustochytrium kinnei n. sp. völlig in der Zeit vom 5. April bis 28. Juni 1965, in der die übrigen Vertreter ihre Blüte hatten, tauchte dann aber im Sommerminimum wieder auf und blieb bis in den September hinein mit nahezu gleicher absoluter Höhe vertreten. Ein erst langsameres, dann stärkeres Abklingen der Vorkommen setzte mit dem ausgehenden Jahre ein.

Unter den entsprechenden Punkten ist jetzt der prozentuale Anteil des Pilzes an der Gesamtpopulation eingetragen. Hier zeigt sich, daß im Februar die gesamte Niedere Pilzpopulation durch Thraustochytrium kinnei n. sp. besetzt war und am 15. März ein relatives Absinken auf $40 \%$ verzeichnet werden konnte, das heißt, daß sich hier bereits wieder die neue Population der übrigen Niederen Pilze aufzubauen begann. In der daran anschließenden Zeit verschwand T. kinnei n. sp. ganz von der Bildfläche, um am 14. Juli im Sommerminimum der übrigen Pilze wieder mit $75 \%$ in Erscheinung zu treten und am 18. August sogar wieder $100 \%$ zu erreichen. Daran anschließend, mit dem Ansteigen der Herbstpopulation der übrigen Pilze hielt sich dieser Vertreter mit $12 \%$ nahezu konstant, um darauf über 3,1 bzw. 1,2\% am 13. Dezember nur noch eine untergeordnete Rolle zu spielen.

Es ergibt sich nun die Frage, ob ein solcher Verlauf der Populationen von Thraustochytrium kinnei $\mathrm{n}$. sp. auch für andere Stellen außerhalb Helgoland charakteristisch ist oder ob wir es hier mit einem speziellen Phänomen aus der Inselnähe zu tun haben. Wir prüften daraufhin die etwa $1,8 \mathrm{sm}$ südlich der Insel gelegene Dauerstation Ansteuerungstonne Helgoland (Abb. 19) und mußten feststellen, daß hier der Pilz überhaupt nur zweimal im Jahr aufgefunden wurde. Zur Zeit des Winterminimums am 15. März mit 18 Pilzeinheiten je Liter Seewasser und 100\% Besatz und am 5. April mit zwei Pilzeinheiten je Liter und $3 \%$ relativer Häufigkeit.

Noch geringer liegen die Häufigkeiten, zieht man die Fluktuationen an der $8 \mathrm{sm}$ NW von Helgoland gelegenen Station 5 sm NW Tonne Nathurn (Abb. 20) hinzu. Hier trat Thraustochytrium kinnei n. sp. nur einmal, am 18. August mit 4 Pilzen je Liter und $13 \%$ relativer Häufigkeit in Erscheinung. Sporadisches Auftreten finden wir, wie Tabelle 1 zeigt, auch im Bereich der Tonne $\mathrm{K}$ und der Tonne D, ersteres unter starkem Brackwassereinfluß in der Wesermündung, letzteres im hochsalinen Gezeitenstrom entlang der Küste. Doch bleiben diese Vorkommen gelegentlicher Natur, ohne daß ein sichtbarer Zusammenhang mit anderen Faktoren vorerst festgestellt werden könnte.

Das gehäufte Auftreten von Thraustochytrium kinnei n. sp. in der Umgebung von Helgoland im ufernahen Gezeitenstrom, jedoch mit einer verhältnismäßig geringen Dichte, weist darauf hin, daß dieser Pilz an das Biotop Helgoland, in der Deutschen Bucht oder auch an ähnliche Biotope im marinen Bereich gebunden sein dürfte und voraussichtlich aus der Uferzone der Insel vorwiegend zu bestimmten Zeiten an das Wasser des Gezeitenstromes als Zoospore abgegeben wird. Dies würde erklären, warum Thraustochytrium kinnei n. sp. niemals ausgesprochene Blüten mit 500 oder 1000 Einheiten im Liter Seewasser gezeigt hat. Vielmehr war das bisher höchste Vorkommen 
im Oktober 1964 mit 55 Pilzen/l im Jahre 1965 nicht wieder erreicht worden. Zieht man jetzt für die Beurteilung seine für Niedere Wasserpilze ungewöhnlich hohe Entwicklungsgeschwindigkeit heran - der ganze Ihnen vorgestellte Entwicklungsgang von der Zoospore bis zum fertigen, schwärmenden Sporangium dauert weniger als 6 Stunden -, so wird eine Bindung an den Gezeitenwechsel noch deutlicher. Diese kann aber nur dann zum Tragen kommen, wenn der Pilz seinen Standort festsitzend in der Uferregion hat.

Tabelle 1

Vorkommen $(+)$ von Traustochytrium kinnei n. sp. in der Zeit vom 1. Februar bis 13. Dezember 1965 an den fünf Dauerstationen: Tonne $\mathrm{K}$ und $\mathrm{D}$ (Wesermündung), Helgoland Ansteuerungstonne, Helgoland Kabeltonne (zwischen Insel und Düne) und 5 sm NW Tonne Nathurn (8 sm NW Helgoland)

\begin{tabular}{|c|c|c|c|c|c|}
\hline \multirow{2}{*}{$\begin{array}{c}\text { Datum der } \\
\text { Entnahme } \\
1965\end{array}$} & \multicolumn{5}{|c|}{ Dauerstationen } \\
\hline & $\begin{array}{c}\text { Tonne } \\
K\end{array}$ & $\begin{array}{c}\text { Tonne } \\
\text { D }\end{array}$ & $\begin{array}{l}\text { Ansteu- } \\
\text { erungstonne } \\
\text { Helgold. }\end{array}$ & $\begin{array}{c}\text { Helgold. } \\
\text { Kabel- } \\
\text { Tonne }\end{array}$ & $\begin{array}{l}5 \mathrm{sm} \text { NW } \\
\text { Tonne } \\
\text { Nathurn }\end{array}$ \\
\hline 1. 2. & - & - & - & - & \\
\hline 25.2 & - & - & - & + & - \\
\hline 15. 3. & - & - & + & + & \\
\hline 5.4 & - & - & + & - & - \\
\hline 12. 4. & - & - & - & - & - \\
\hline $17 . / 18.4$ & - & - & - & - & - \\
\hline 24.5. & - & - & - & - & - \\
\hline $8,6$. & - & + & - & - & - \\
\hline 28. 6. & - & - & - & - & \\
\hline 14. 7. & - & - & - & + & - \\
\hline 18.8 & + & - & - & + & + \\
\hline 15.9. & - & - & - & + & - \\
\hline 4.10 & - & + & - & + & - \\
\hline 27.10 & - & - & - & + & - \\
\hline 15.11. & + & - & - & + & - \\
\hline 13.12. & + & - & - & + & - \\
\hline
\end{tabular}

Um hierfür Anhaltspunkte zu gewinnen, führten wir zu verschiedenen Zeiten gelegentliche Entnahmen von Wasser aus der Zone des Spülsaumes um Helgoland durch, beköderten sie mit Pollen und stellten fest, daß hiervon mindestens $80 \%$ den Pilz enthielten. Sogar alle Entnahmen waren besetzt, gaben wir dem Wasser kleine Stücke von Thalli verschiedener Algen aus der Uferzone wie Enteromorpha, Porphyra, Fucus, auch angetriebenes Material von Delesseria und Laminaria zu, ohne daß man bei mikroskopischer Kontrolle gleichgroßer Stücke gleicher Entnahme irgendeinen Pilzbesatz oder Befall nachweisen konnte. Dies muß vorerst als weiteres Indiz dafür angesehen werden, daß Thraustochytrium kinnei an die Pflanzengesellschaften der Uferzone gebunden ist, ohne daß bisher die Frage seiner Biologie, welcher Art die Bindung ist, näher geklärt werden konnte. Jedoch ist wahrscheinlich, daß hierfür ähnliche Verhältnisse gelten, wie sie Sparrow (1936) für Thraustochytrium proliferum und Коваулаs \& Oокuвo (1953) für Thraustochytrium globosum angeben, nämlich, daß diese Pilze schwache Algenparasiten oder Saprophyten sind, deren Rolle im Haushalt des Biotopes noch wenig geklärt ist. Damit steht dieser Pilz offensichtlich im Gegensatz zu anderen Thraustochytriaceae, die wir bei unseren Untersuchungen in der 
Wesermündung und der Deutschen Bucht ständig, nur mit wechselnden Besatzzahlen finden und die die Ihnen in den Diagrammen aufgezeigten Massenvermehrungen vollziehen. Wir müssen darüber hinaus annehmen, daß einige von ihnen zu einer fest umrissenen Mykoflora planktontisch lebender Pilze gehören, welche wir geneigt sind, als Hochseepilze anzusprechen.

\section{ZUSAMMENFASSUNG}

1. Der aus dem Gezeitenstrom zwischen Insel und Düne Helgoland erstmalig isolierte Pilz Thraustochytrium kinnei $\mathrm{n}$. sp. zeichnet sich durch besonderen Habitus und Entwicklungsgang von den bisher beschriebenen Arten des gleichen Formenkreises ab.

2. Hierdurch bot er sich als leicht zu identifizierendes Objekt für die mitgeteilten Untersuchungen zur Ökologie mariner Niederer Pilze an.

3. Wir verfolgen die Vorkommen Niederer Pilze der Gattungen Thraustochytrium, Schizochytrium und Dermocystidium an fünf Dauerstationen in der Wesermündung und der Deutschen Bucht. Hier zeigte sich, daß T. kinnei nur bei Helgoland in den Monaten Juli bis Dezember mit einiger Sicherheit aufzufinden war, während er im April bis Juni 1965 fehlte.

4. Aus dem Verhalten von T. kinnei und dem Fehlen einer typischen Gradation müssen wir schließen, daß es sich hierbei um einen Uferbewohner handelt, der in Zoosporenform im Gezeitenstrom mitgeführt wird.

5. Weitere Untersuchungen zur Physiologie des Pilzes sind im Gange und sollen Aufschluß über seine spezifischen Ansprüche geben.

Die mitgeteilten Untersuchungen wurden durch die Deutsche Forschungsgemeinschaft maßgeblich gefördert. Der Deutschen Forschungsgemeinschaft sei hierfür vielmals gedankt. Ferner danke ich Frau M. Höhnk, Fräulein I. Harrigreld und Frau R. EhLKen für technische Hilfe sowie Herrn Dr. med. E. Schaefer, Göttingen, für die Korrektur der lateinischen Diagnose.

\section{ZITIERTE LITERATUR}

Gaertner, A., 1954. Uber das Vorkommen niederer Erdphycomyceten in Afrika, Schweden und an einigen mitteleuropäischen Standorten. Arch. Mikrobiol. 21, 4-56.

- 1964. Elektronenmikroskopische Untersuchungen zur Struktur der Geißeln von Thraustochytrium sp. Veröff. Inst. Meeresforsch. Bremerh. 9, 25-30.

- 1966. Vorkommen, Physiologie und Verteilung ,Mariner niederer Pilze' (Aquatic Phycomycets). Veröff. Inst. Meeresforsch. Bremerh. (Sonderbd) 2, 221-236.

GoldSTEIN, S., 1963. Morphological variation and nutrition of a new monocentric marine fungus. Arch. Mikrobiol. 45, 101-110.

- 1966. Biology of a problematic marine fungus, Dermocystidium sp. Arch. Mikrobiol. 53, $1-11$.

HARDER, R., 1948. Uber das Vorkommen niederer Phycomyceten in deutschen Böden. Nachr. Akad. Wiss. Göttingen (Math.-phys. Kl.) 1948, 5-7.

Höнnk, W., 1955. Niedere Pilze von Watt und Meeresgrund (Chytridiales und Thraustodhytriaceae). Naturwissenschaften 42, 348-349.

- 1957. Fortschritte der marinen Mykologie in jüngster Zeit. Naturw. Rdsch., Stuttg. 11, 39-44. 
Jornson, T. W., 1957. On the marine Phycomycete Thraustochytrium proliferum. Trans. Br. mycol. Soc. 40, 292-294.

Kobayasi, Y. \& Ookubo, M., 1953. Studies on the marine Phycomycetes. Bull. natn. Sci. Mus., Tokyo 33, 53-65.

SparRow, F. K., Jr., 1936. Biological observations on the marine fungi from Woods Hole waters. Biol. Bull. mar. biol. Lab., Woods Hole 70, 236-263.

- 1960. Aquatic phycomycetes. 2nd ed. Univ. of Michigan pr., Ann Arbor, 1187 pp.

\section{Diskussion im Anschluß an den Vortrag GAERTNER}

KINNE: Ich möchte Ihnen, lieber Herr Kollege Gaeri'NeR, zunächst sehr herzlich Dank sagen dafür, daß Sie diesen interessanten Pilz nach mir benannt haben (schade, daß er so selten ist!). Die bisher erarbeiteten Informationen lassen starke jahreszeitliche Abundanzfluktuationen erkennen. Haben Sie Vermutungen hinsichtlich der möglichen Ursachen dieser Fluktuationen?

GAERTNER: Über die möglichen Ursachen der Abundanzfluktuationen können wir zur Zeit noch nichts sagen. Wir müssen annehmen, daß das Nahrungsangebot neben anderen Faktoren dabei die Hauptrolle spielt, besonders an gelösten Stoffen für die "planktischen" Formen. Die Fluktuationen sollten in Zusammenhang oder als Folge von Zusammenbrüchen planktischer Organismen in Seewasser gesehen werden.

KINNE: Wovon ernährt sich der Pilz in der freien Natur? Können Sie über seine Rolle im Okosystem etwas aussagen?

Gaertner: Die Frage nach der natürlichen Nahrung des Pilzes können wir zur Zeit noch nicht beantworten. Wir haben in unseren Planktonfängen noch keinen unter natürlichen Bedingungen gewachsenen Pilz gefunden, obgleich wir seine Existenz im Köderverfahren nachgewiesen haben. Sicher ist, daß T. kinnei in Algen gehäuft vorkommt; vielleicht ist er ein schwacher Parasit. Durch mikroskopische Untersuchung läßt er sich nicht ohne weiteres nachweisen, wahrscheinlich wegen seiner sehr hyalinen Struktur; er wird übersehen oder mit anderen, etwa tierischen Organismen und deren Cysten verwechselt.

HöнNк: In der Systematik sind die Thraustochytrien immer nahe den Saprolegnien placiert worden, denn in beiden Familien bilden die Mitglieder zweizeilige Zoosporen. In den lange bekannten Saprolegniaceae spielt aber neben der Morphologie der Sporen auch ihr Verhalten eine Rolle; und zwar bei der Aufgliederung in Gattungen. Immer beginnt die individualisierte seitlich begeißelte Zoospore mit einem Ruhestadium, das sie in einer selbstgebildeten Membran verbringt. Nach dem Ausschlüpfen beginnt ihre Schwärmzeit. Bei Thraustochytrium kinnei beobachteten Sie die seitlich begeißelte Zoospore gleich aus dem Sporangium und ohne Häutung davoneilend. Dies Verhalten ist für die Saprolegnien nicht bekannt. Ist diese Erscheinung die systematische Scheide zwischen den Saprolegniaceae und den Thraustochytriaceae?

GAERTNER: Ursprünglich war die Trennung zwischen Saprolegniaceae und Thraustochytriaceae nach dem Verhalten ihrer Zoosporen erfolgt. Es zeigte sich aber, daß hier innerhalb der $\mathrm{Fa}$ milie zwei Pole vorhanden sind: Thraustochytrium proliferum mit Restkörper und Zoosporen mit vorhergegangenem Ruhestadium und Thraustochytrium globosum ohne Restkörper und ohne vorheriges Ruhestadium. Hier stellt Thraustochytrium kinnei das Bindeglied dar: Ausgebildeter Restkörper bei Zoosporen ohne ein Ruhestadium.

HöHNK: Den Basalteil unterhalb des Sporangiums haben wir bei einer nicht bestimmten Art noch deutlicher, als Ihr Dia zeigte, ausgebildet gesehen. Er könnte Hyphencharakter haben oder eine gestauchte Hyphe sein. Darum die Frage: Gehören die Thraustochytriaceae mit den Saprolegniaceae in eine phylogenetische Reihe, sind die Thraustochytrien von den Saprolegnien abzuleiten?

GAERTNER: Als Denkschema ist die Auffassung, ihr Basalteil und der Restkörper bei den Thraustochytrien sei eine gestauchte Hyphe, nützlich. Schwierig im Sinne einer phylogenetischen Ableitung ist nur die Entstehung des Restkörpers im Innern des Sporangiums. Wäre er eine Hyphe, so müßte diese primär vorhanden sein und an dieser, wie in Ihrem Falle das Sporangium entstehen. Zur Frage der Klärung der Genese des Restkörpers bedarf es noch eingehender zytologischer Studien. 